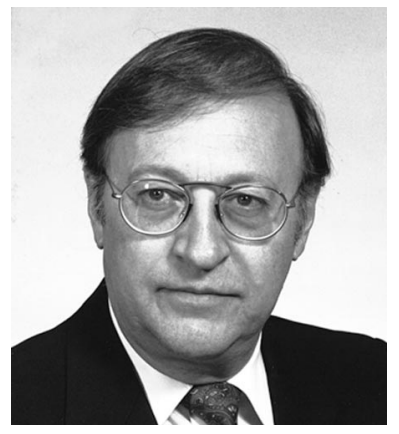

\section{Why do we use keywords?}

In my mind, keywords are used for classification, rationalization, and for helping anyone find out what is going on in a specific area.

The first keyword that authors might think of is probably the one that will help characterize the area in which their paper fits best. It might also be an area where they want to see their results used. This is their decision.

We ask for four or five keywords, which gives authors a chance to find other keywords that will confine the search of someone else who may want to research the same subject.

Using one keyword in a search yields many papers and opens up a lot of possibilities. A second keyword also yields many papers, although it is a slightly reduced number, and gives another set of possibilities. We can go on and on like that with all of the keywords, refining our classification until there is a relatively small number of papers fitting in this series.

I do not want to do any combinatory analysis and statistics, but if we imagine for a moment that this classification is not done properly, we will end up with a huge number that will render further classification impossible.

It was good when everyone thought to keep the system simple. There was a time when good managers were working on the KISS system (Keep it simple, stupid!) to be sure that everyone would understand what was going on.

Nowadays, we have computers that are able to process large databases. This makes us confident, and we are reaching a point where we stop thinking about simplification since the system can process everything we put in it. However, by doing this we forget those who do not have access to the same computer systems and who have to search in an old-fashioned manner. Those individuals will not be able to process such a large amount of information and will not be able to trace the work already done and published in their subject area.

After my recent editorial about referencing, someone wrote to me and said that I was much too inclined to think that everyone has access to computers for reference searches. I was shocked by this and started to think about the fact that there are places where computerized searches are not yet available. This person was right since I was thinking like a North American who has access to computers without problems. It is even worse for keywords.

My experience is expanding with the number of papers I see as Editor. After that remark from one of our readers, I started verifying keywords used by authors. I found that many do not characterize their work, but rather use only very narrow keywords that others may not think of. I suspect that many researchers will not be able to trace down papers that could potentially be of interest to them.

Consequently, the problem created by this difficulty of tracing down papers will emphasize the fact that I wanted to make in my recent editorial about referencing. A lack in both finding references and not looking for references contributes to an increase in the number of inappropriate papers that are republishing work that has already been done. I believe that one part of the republication problem comes from the lack of access to proper sources due to a lack of well-defined keywords.

I suspect that many readers will not fully agree with my point of view, but I hope that many will think of a way of defining keywords that would really help everyone in their search for simplicity and efficiency.

Looking forward to reading your comments.

Roger A. Lessard Editor 


\section{Rudolf Kingslake Medal and Prize}

The Rudolf Kingslake Medal and Prize is awarded annually in recognition of the most noteworthy original paper to appear in Optical Engineering on theoretical or experimental aspects of optical engineering. The 1999 Rudolf Kingslake Medal and Prize is awarded to Robert D. Fiete for his paper entitled "Image quality and $\boldsymbol{\lambda} \mathbf{N} / \mathbf{p}$ for remote sensing systems" which appeared in the July 1999 issue. This paper was selected by the Kingslake Award Committee as an excellent analysis paper that will add "significantly to the community understanding of how to design imaging systems.'

\section{Rudolf Kingslake Medal and Prize-Past Recipients}

1974 Irving R. Abel and B. R. Reynolds

1975 J. M. Burch and C. Forno

1976 Richard E. Swing

1977 David B. Kay and Brian J. Thompson

1978 Norman J. Brown

1979 J. R. Fienup

1980 G. Ferrano and G. Hausler

1981 Robert A. Sprague and William D. Turner

1982 David M. Pepper

1983 James R. Palmer

1984 Gene R. Gindi and Arthur F. Gmitro

1985 Armand R. Tanguay, Jr.

1986 Arthur D. Fischer, Lai-Chang Ling, John N. Lee, and Robert C. Fukuda

1987 Chris P. Kirk

1988 Ares J. Rosakis, Alan T. Zehnder, and Ramaratnam Narasimhan

1989 Pochi Yeh, Arthur Chiou, John Hong, Paul H. Beckwith, Tallis Chang, and Monte Khoshnevisan

1990 Paul R. Prucnal and Philippe A. Perrier

1991 Brian E. Newman

1992 Aden B. Meinel and Marjorie P. Meinel

1993 Harvey M. Phillips and Roland A. Sauerbrey

1994 Jose M. Sasian

1995 Arnold Daniels, Glenn D. Boremann, Alfred D. Ducharme, and Eyal Sapir

1996 Pär Kierkegaard

1997 Gleb Vdovin, Simon Middlehoek, and Pasqualina M. Sarro

1998 Russell C. Hardie, Kenneth J. Barnard, John G. Bognar, Ernest E. Armstrong, and Edward A. Watson 